\title{
Comprehensively Identifying the Key tRNA-Derived Fragments and Investigating Their Function in Gastric Cancer Processes
}

This article was published in the following Dove Press journal: OncoTargets and Therapy

\author{
Xiaolin Dong ${ }^{1,2, *}$ \\ Xirui $\mathrm{Fan}^{3, *}$ \\ Xiaoxue $\mathrm{He}^{3}$ \\ Sijin Chen $^{3}$ \\ Weikang Huang ${ }^{3}$ \\ Jianpeng $\mathrm{Gao}^{2,3}$ \\ Yun Huang ${ }^{2,3}$ \\ Hui Wang ${ }^{2,3}$
}

'Department of Neurology, The Affiliated Yan'An Hospital of Kunming Medical University, Kunming 65005I, Yunnan, People's Republic of China; ${ }^{2}$ Key Laboratory of Tumor Immunological Prevention and Treatment of Yunnan Province, Kunming 65005I, Yunnan, People's Republic of China; ${ }^{3}$ Department of Gastroenterology, The Affiliated Yan'An Hospital of Kunming Medical University, Kunming 65005I, Yunnan, People's Republic of China

*These authors contributed equally to this work
Correspondence: Yun Huang; Hui Wang The Affiliated Yan'An Hospital of Kunming Medical University, No. 245 Renmin East Road, Kunming 65005I, Yunnan, People's Republic of China

Email 9542287@qq.com;

weihool@163.com
Purpose: Gastric cancer (GC) is the second leading cause of cancer-related deaths worldwide. tRNA-derived fragments (tRFs) have been identified as potential biomarkers and cancer therapeutic targets. However, the influence of tRFs on GC remains unknown. The key tRFs were researched in vitro function and mechanism.

Patients and Methods: Here, differentially expressed tRFs between GC and paracancerous tissues were identified by small RNA sequencing, and the role of key tRF was evaluated in vitro. Results: Eight tRFs were significantly differentially expressed between GC tissues and adjacent tissues: five were significantly upregulated and three were downregulated in GC tissues. The results of target gene prediction and functional enrichment analysis showed that tRFs with different expressions were mainly involved in cell adhesion and connection, cell migration, wingless-type (Wnt), mitogen-activated protein kinase (MAPK), and cancer signaling pathways. Quantitative real-time polymerase chain reaction (qRT-PCR) indicated that the expression of tRF-24-V29K9UV3IU and its target genes (CCND2, FZD3, and VANGL1) in GC tissues and cells was decreased compared with those in the control group. Importantly, overexpression of tRF-24-V29K9UV3IU inhibited cell proliferation, migration and invasion, while promoted cell apoptosis of GC cells.

Conclusion: This study suggests that tRF-24-V29K9UV3IU may hinder GC tumor progression by inhibiting cell proliferation, migration, invasion, while promoting cell apoptosis by regulating the Wnt signaling pathways.

Keywords: gastric cancer, tRF, invasion and migration, cell apoptosis, tRF-24V29K9UV3IU

\section{Introduction}

Gastric cancer (GC) has the second highest morbidity and mortality rate, next to lung cancer. ${ }^{1}$ Most of the patients were in the advanced stage of GC at the time of diagnosis, and GC cells had metastasized. A simple radical surgical resection could not completely cure the disease, and the recurrence rate is high. ${ }^{2}$ With the increasing trend of the aging population in China, the social burden caused by GC will more likely increase. Gastroscopy, which is an invasive procedure, is an important method for the early diagnosis of GC. The sensitivity and specificity of tumor biomarkers used in clinical practice should be improved. ${ }^{3}$ Currently, there are no new diagnostic markers and therapeutic targets for GC treatment.

Transfer RNA (tRNA) is usually 70-90 nucleotides long, and its job is to convert the codon information carried by messenger RNA (mRNA) into amino acid sequence 
information on the new protein. tRNA sources of non-coding RNA (ncRNA), nucleotide composition, and the size of molecules with specific physiological functions, ${ }^{4,5}$ can be roughly divided into two categories: tRNA halves (tiRNAs) and tRNAderived fragments (tRFs).

tRFs are short ncRNAs of about 16-40 nucleotides in length. ${ }^{6}$ They are involved in a variety of physiological and pathological processes, such as viral infection, oxidative stress, and tumors. ${ }^{7-9}$ tRFs can function in a variety of regulatory forms, such as inhibiting mRNA translation by miRNA; competitively binding to RNA-binding proteins to regulate the stability of other genes; ${ }^{10}$ replacing the translation initiation factor eIF4G binding to mRNA; inhibiting the protein translation process, working as the reverse transcription primer of the RNA genome of retrovirus; and participating in the assembly of precursor ribosomal RNA (rRNA) splicing complex. ${ }^{11}$ However, profiles of tRFs in GC patients remain unknown. The function of tRFs and their mechanisms of influencing GC processes will become a meaningful new aspect under investigation.

Previous studies have shown that cancer cells escape from their hypoxic environment through the process of tumor metastasis, and the expression level of tRFs is significantly increased due to hypoxic stress. ${ }^{12}$ Therefore, the regulatory effect of tRF on tumor invasion and metastasis has received extensive attention in recent years. Abnormal expression of multifarious tRF has been found in multifarious tumor cell lines. ${ }^{13}$ tRF-1001, a tRF-1 derived from the precursor of tRFSer, was found to be highly expressed in various tumor cell lines, and it promotes cell proliferation. Knocking down tRF-1001 will result in the inhibition of cell proliferation. ${ }^{4}$ Olvedy et al revealed that tRF315/tRF-544 ratio may be a potential marker of prostate cancer progression. ${ }^{14}$ Zhou et al discovered that tRF5-Glu promotes the proliferation of ovarian cancer cells by regulating the expression of BCAR3, and it can be used as a molecular target for the treatment of ovarian cancer. ${ }^{15}$ tRF/miR-1280 inhibits tumorigenesis in colon cancer by activating the Notch signaling pathway. ${ }^{16}$ Goodarzi et al showed that tRFs inhibited tumor progression by competing for the YBX1 binding site and reducing the stability of breast cancer gene transcription products. ${ }^{17}$ These studies have shown that tRF plays a key role in tumorigenesis and is expected to be used as a tumor diagnostic or prognostic marker or even a tumor therapeutic target. However, the role of tRFs in GC remains unknown.

This study aimed to determine the GC-associated tRFs and explore the possibility of detecting essential tRF in GC cancer cells. Small RNA sequencing was used to analyze the GC and paracancerous tissue samples from our study patients. Moreover, the influences of differently expressed tRFs on GC cells were evaluated using the CCK-8 assay, flow cytometry, and transwell migration assay.

\section{Patients and Methods GC Patients and Data Collection}

This study included GC tissues from 38 patients who underwent surgical resection. Samples of adjacent non-cancerous tissues were categorized as the control group. Small RNA sequencing was performed on the $\mathrm{GC}$ tissues and adjacent tissues of three GC patients. Quantitative real-time polymerase chain reaction (qRT-PCR) was used to verify differentially expressed tRF in GC tissues and the adjacent tissues of 10 GC patients. Further validation of the key tRF in 28 patients with GC tissues and adjacent tissues are required. No pathological changes in the tumor were observed in any of the patients' other organs before surgery, and chemoradiotherapy and immunotherapy were not administered to treat the tumor. Liquid nitrogen was used to freeze the samples immediately after collection. This study was approved by the ethics committee of Kunming Medical University. All patients provided written informed consent.

\section{Small RNA Sequencing}

Small RNA sequencing of GC and the adjacent tissues from three patients was performed. Total RNA was extracted with TRIzol (Invitrogen, USA). After performing NanoDrop spectrophotometry and agarose gel electrophoresis, small RNAs between 18 and 30 nucleotides (nt) long were separated and purified using 15\% TBE polyacrylamide gel (Invitrogen). The small RNA library construction kit (NEB, USA) was used to construct the sequencing library. Agilent 2100 (Agilent Technologies) was used to detect the quality of the small RNA library construction, and the library was sequenced using the Illumina Hiseq 2500 (Illumina, USA).

\section{Bioinformatic Analysis}

The raw sequencing data were analyzed using FASTQC, including the quality distribution of nucleotides, positionspecific sequencing quality, GC content, the proportion of PCR duplicates, and k-mer frequency. First, small RNA expression was obtained from the microRNA database by relatively clean reading. The undefined sequences were compared with those obtained from the genomic tRNA 
database to determine the level of tRF expression. The tRFs obtained from the tRF database were compared with those obtained from the tRF MINTbase, and corresponding suggestions were raised. The differentially expressed tRFs, which were obtained by analyzing the small RNA expression from cancer tissues and adjacent tissues were identified based on $\mid \log 2$ fold change $\mid>1$, FDR $<0.05$. Target genes of different tRFs were predicted using miRanda and RNAhybrid. The Gene Ontology (GO) and Kyoto Encyclopedia of Genes and Genomes (KEGG) enrichment analyses of target genes were carried out to preliminarily analyze the functions of tRF with different expressions.

\section{Real-Time PCR}

Five tRFs with high fold differences and high expression levels were selected for further verification by real-time PCR. Total RNA in cancer tissues and paracancerous tissues from 38 patients with GC were extracted using TRIzol (Invitrogen), and cDNA was reverse-transcribed using a high-quality total RNA reverse transcriptional kit (Thermo Bio). qRT-PCR was performed using the ABI Q6 (Applied Biosystems Inc., USA). According to the manufacturer's instructions, the primers showed in Table 1.

The PCR amplification was performed using the following parameters: pre-denaturation at $95^{\circ} \mathrm{C}$ for $10 \mathrm{~min}$, $95^{\circ} \mathrm{C}$ for $15 \mathrm{sec}$, and $60^{\circ} \mathrm{C}$ for $60 \mathrm{sec}$ for 45 cycles using ABI 7500 real-time PCR system (Applied Biosystems, Foster City) and solution curve stage at $95^{\circ} \mathrm{C}$ for $10 \mathrm{sec}$, $60^{\circ} \mathrm{C}$ for $60 \mathrm{sec}$, and $95^{\circ} \mathrm{C}$ for $15 \mathrm{sec}$. The relative expression level of tRFs was analyzed using the threshold cycle (Ct) method $\left(2^{-\Delta \Delta C t}\right)$, and the endogenous reference gene was U6.

\section{Cell Culture and Transfection}

BGC-823 and MKN-45 cells, which were provided by the cell bank of the Chinese Academy of Sciences (Shanghai, China), were cultured under ATCC recommended conditions and supplemented with 10\% fetal calf serum. The medium was changed every other day, and the cells were passaged every 3-4 days. The cells in the exponential phase were selected for further experiments. The cultured cells were uniformly seeded in 6 -well cultured plates at a density of $3 \times 10^{5} / \mathrm{mL}(2 \mathrm{~mL}$ per well), and cell growth was observed under an
Table I Primer Sequences

\begin{tabular}{|l|l|}
\hline tRF Name & Sequence(5'-3') \\
\hline U6-F & CGATACAGAGAAGATTAGCATGGC \\
U6-R & AACGCTTCACGAATTTGCGT \\
tRF-21:tsRNA-02257-F & GCGACGCAATTCCTCTTCTT \\
tRF-21:tsRNA-02257-R & AGTGCGTGTCGTGGAGTCG \\
tRF-22:tsRNA-19528-F & GCAGCATTGGTGGTTCAGTG \\
tRF-22:tsRNA-19528-R & AGTGCGTGTCGTGGAGTCG \\
tRF-24:tsRNA-18946-F & GACTACACTAGGATGGGGTGTGATA \\
tRF-24:tsRNA-18946-R & AGTGCGTGTCGTGGAGTCG \\
tRF-28:tsRNA-14694-F & GACGATCGCATGGGTGGTT \\
tRF-28:tsRNA-14694-R & CTCTTCCGATCTGAGAATTCTACCA \\
tRF-30:tsRNA-06I26-F & CCGACGATCAGCATGGGTG \\
tRF-30:tsRNA-06I26-R & TTCCGATCTCGAGAATTCTACCA \\
\hline
\end{tabular}

inverted microscope. When the cells reached 60-70\% confluence, they were detached using $0.25 \%$ trypsin (Gibco; Thermo Fisher Scientific, Inc.). tRF-24 mimics, NC mimics, and scramble (RiboBio, Guangzhou, China), were transiently transfected using Lipofectamine 2000 (Invitrogen, Carlsbad, CA, USA) according to the manufacturer's instructions.

\section{CCK-8}

Cell proliferation was detected by the CCK-8 assay. After 48 h of transfection using Lipofectamine 2000 reagent, BGC823 cells (Cell Bank of Shanghai Institute of Cell Biology, China) were digested into a single cell suspension and counted using a Bright-Line Counting Chamber (1492, Hausser Scientific). Each group of cells was seeded in 96well plates (3599, Corning, NY, USA) at a density of $2 \times 10^{4}$ cells/mL per well, with six compound wells for each sample, and 100 microns of sterile water or phosphate buffer saline (PBS) was added to the edge wells. The cells were cultured and incubated in a $100 \% \mathrm{CO}_{2}$ incubator at $37^{\circ} \mathrm{C}$. After 0,24 , 48,72 , and $96 \mathrm{~h}$ of cell culture, $10 \mu \mathrm{L}$ of CCK-8 (Shanghai Biyuntian Bio-Technology Co., Ltd.) was added at each detection time point. After $1 \mathrm{~h}$, the optical density was measured at $450 \mathrm{~nm}$ with an enzyme marker (Infinite M1000, TECAN).

\section{Flow Cytometry}

Flow cytometry was used to detect the apoptosis of GC cells in the transfection and NC groups. Cells in the logarithmic growth phase were collected and counted. The cells were inoculated in 6-well plates with 
$(1-5) \times 10^{5}$ cells/well, incubated at $37^{\circ} \mathrm{C}$, and cultured overnight under $5 \% \mathrm{CO}_{2}$, to ensure that the cells were adherent to the wall. The next day, the culture medium was changed, and a $2 \mathrm{~mL}$ cell culture medium containing different concentrations of theanine was added to each well, and the conventional culture was continued. The liquid was changed every $24 \mathrm{~h}$ using the same method. After $48 \mathrm{~h}$ of drug treatment, the cells were digested with ethylenediaminetetraacetic acid (EDTA)free trypsin and centrifuged by a flow tube at $1000 \mathrm{rpm}$ for $5 \mathrm{~min}$ to discard the supernatant. The cells were washed with cold PBS three times, and the supernatant was discarded by centrifugation. Using the Annexin V-FITC Apoptosis Detection Kit, the cells were harvested, washed, and stained at room temperature with 10 microns of Annexin $\mathrm{V}$ and with 5 microns of propidium iodide (PI) for $15 \mathrm{~min}$ at room temperature. Flow cytometry (Epics Altra II; Beckman Coulter, Fullerton, CA, USA) was used to measure apoptotic cells.

\section{Transwell}

A transwell assay was used to detect the invasion and migration of GC cells in the transfected and NC groups. After harvesting $\mathrm{BGC}-823$ cells, the medium containing $10 \%$ serum was used to continue incubating the cells in the $\mathrm{CO}_{2}$ incubator (Forma Series II Water Jacket, Thermo) for $24 \mathrm{~h}$ at room temperature. The chamber was then removed. The cells inside the chamber were stained with crystal violet dye solution (C0121, Bi Yuntian) at room temperature for $30 \mathrm{~min}$ and gently rinsed with water severally. The chamber was subsequently removed, and the liquid in the upper chamber was absorbed. Next, the cells on the surface of the upper chamber membrane were carefully removed using a wet cotton swab; the membrane was carefully peeled off with a small forceps, and the bottom surface was dried. The cells were transferred to a glass slide (F518101, raw work), and the slide was sealed with neutral gum (E675007, aging). The three random fields were assessed under a microscope (Nikon), and the results were counted.

\section{Statistical Analysis}

Each experiment was repeated three times. All statistical analyses were performed using the SPSS package (version 22.0) and GraphPad prism software (version 8.0.1), and the results are shown as mean \pm SD. Significant differences between GC tissue and paracancer tissue groups were determined by unpaired Student's $t$-test, and
$\mathrm{P}$ values $<0.05$ were considered significant. Data are expressed as mean \pm standard deviation (SD).

\section{Results}

\section{Mapping of Small RNA Sequencing in Different Samples}

More than 14,154,494 clean reads were generated in each library. After filtering, approximately $87 \%$ of the reads survived. On average, 21,302,635, 16,946,054, 18,653,837, $17,849,860, \quad 19,500,668, \quad 18,782,297, \quad 20,613,103$, $17,064,328,14,154,494$, and 18,519,277 clean reads were obtained for each library of N514, T514, N519, T519, N521, T521, N561, T561, N586, and T586, respectively (Table S1). More than $39.9 \%$ of the total clean reads were mapped to the miRNA database (Table S2). The average potential piRNAs in all the samples was approximately 59,306 , with a maximum of 118,054 and a mapping rate of $5.4 \%$, while the minimum was 25,514 with a mapping rate of $3 \%$ (Table S3). The maximum mapping rate of known piRNA was $7.7 \%$, while the minimum was $2.1 \%$. The maximum known piRNA unique mapping was 1823 in each sample (Table S4). The tsRNA mapping rate of each sample was greater than $73 \%$, and the unique mapped rate was less than $1 \%$ (Table S5).

\section{Analysis of Differentially Expressed tRFs Between GC Tissues and Adjacent Tissues}

We analyzed the small RNAs in 3 pairs of GC tissues and paracancerous tissues by small RNA sequencing. We found 32 differentially expressed small RNAs in GC tissues compared with adjacent tissues, including 24 differentially expressed miRNAs and 8 differentially expressed tRFs (Figure 1A and 1B). Five tRFs were significantly upregulated in $\mathrm{GC}$ tissues, while the expression levels of three tRFs (including tRF-24-V29K9UV3IU) were significantly downregulated (Figure 1C).

\section{GO and KEGG Analyses of the Differentially Expressed tRFs}

We employed miRanda (http://www.microrna.org/microrna/ home.do) and RNAhybrid (http://bibiserv.techfak.unibielefeld.de/rnahybrid/) to predict the target genes of the differentially expressed tRFs and performed $\mathrm{GO}$ and KEGG analyses for these target genes. GO analysis showed that these differential tRF target genes were mainly involved 
A

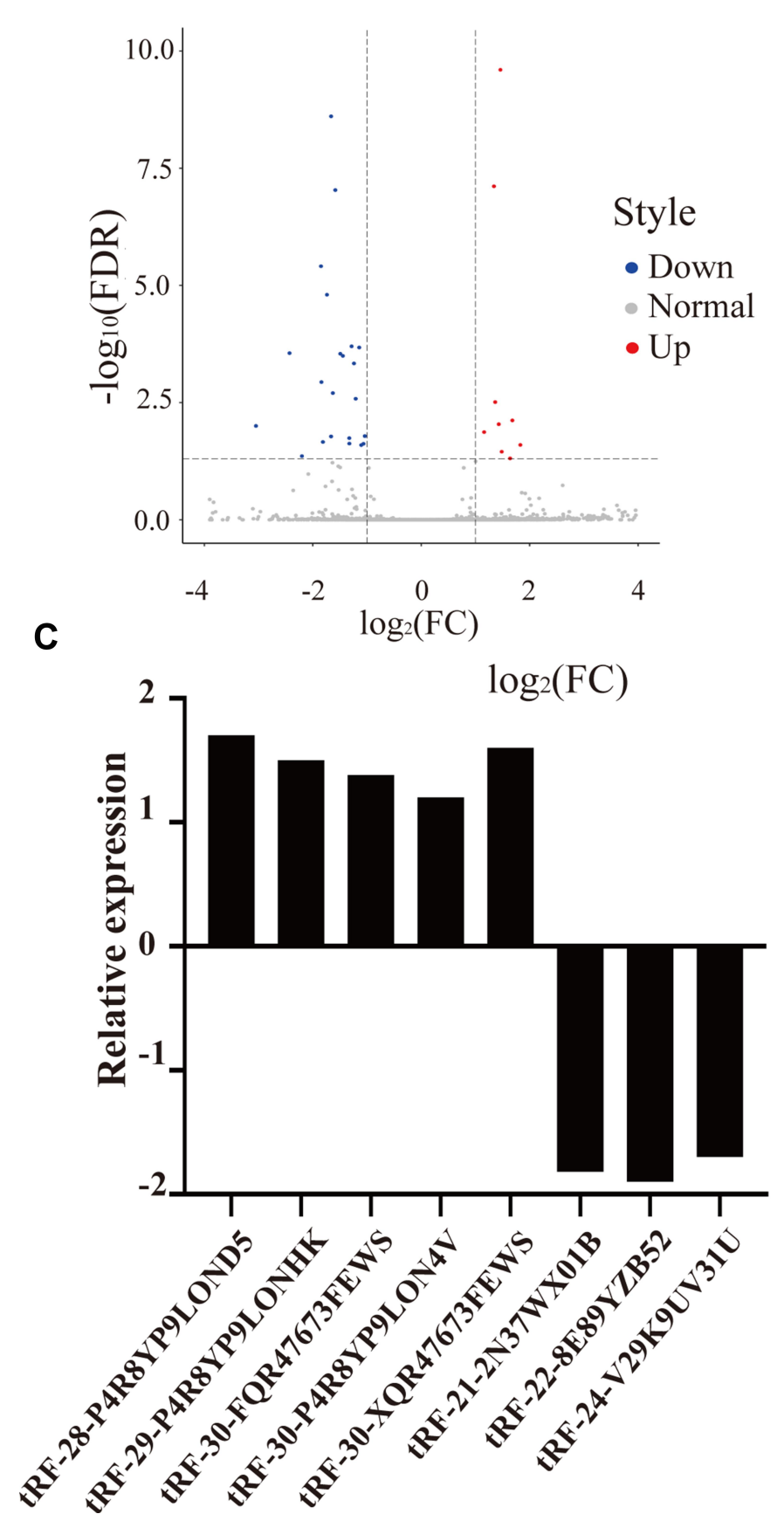

B

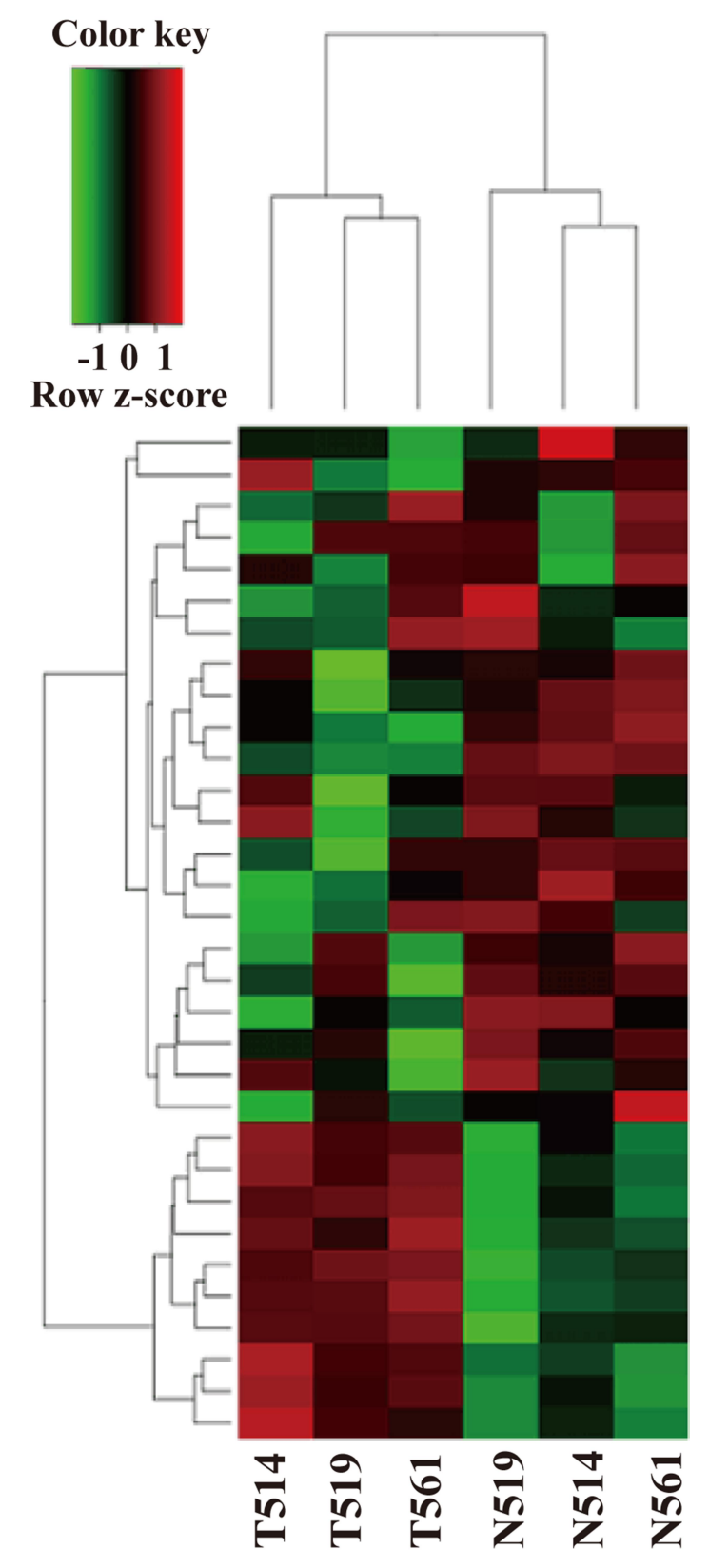

Figure I Small RNA sequencing analysis of the differential expression of tRFs between GC tissues and adjacent tissues of 3 gastric cancer (GC) patients. (A) Volcano plot displayed differential expression of small RNAs between GC tissues and adjacent tissues of $3 \mathrm{GC}$ patients; red and blue dots represent expression upregulation and downregulation, respectively, while gray dot indicates no significant change. (B) Heatmap demonstrated differential expressions of miRNAs and tRFs between GC tissues ( $T$ ) and adjacent tissues (N) of 3 GC patients (red and green dots indicate upregulation and downregulation in GC tissues, respectively)). (C) Quantitative reverse transcription polymerase chain reaction (qRT-PCR) showed 8 tRFs differentially expressed in 10 GC patients; positive numbers indicate upregulation and negative numbers indicate downregulation.

in cell adhesion, cAMP catabolism, cell migration, cell junction, and other life activities (Figure 2A). KEGG enrichment analysis revealed that $\mathrm{tRF}$ target genes were mainly involved in cancer proteoglycan synthesis, stem cell differentiation regulation, focal adhesion, MAPK, Wnt, and calcium signaling pathways (Figure 2B). 
A

Molecular function
Cellular component
Biological process

\section{-log(p-value)}

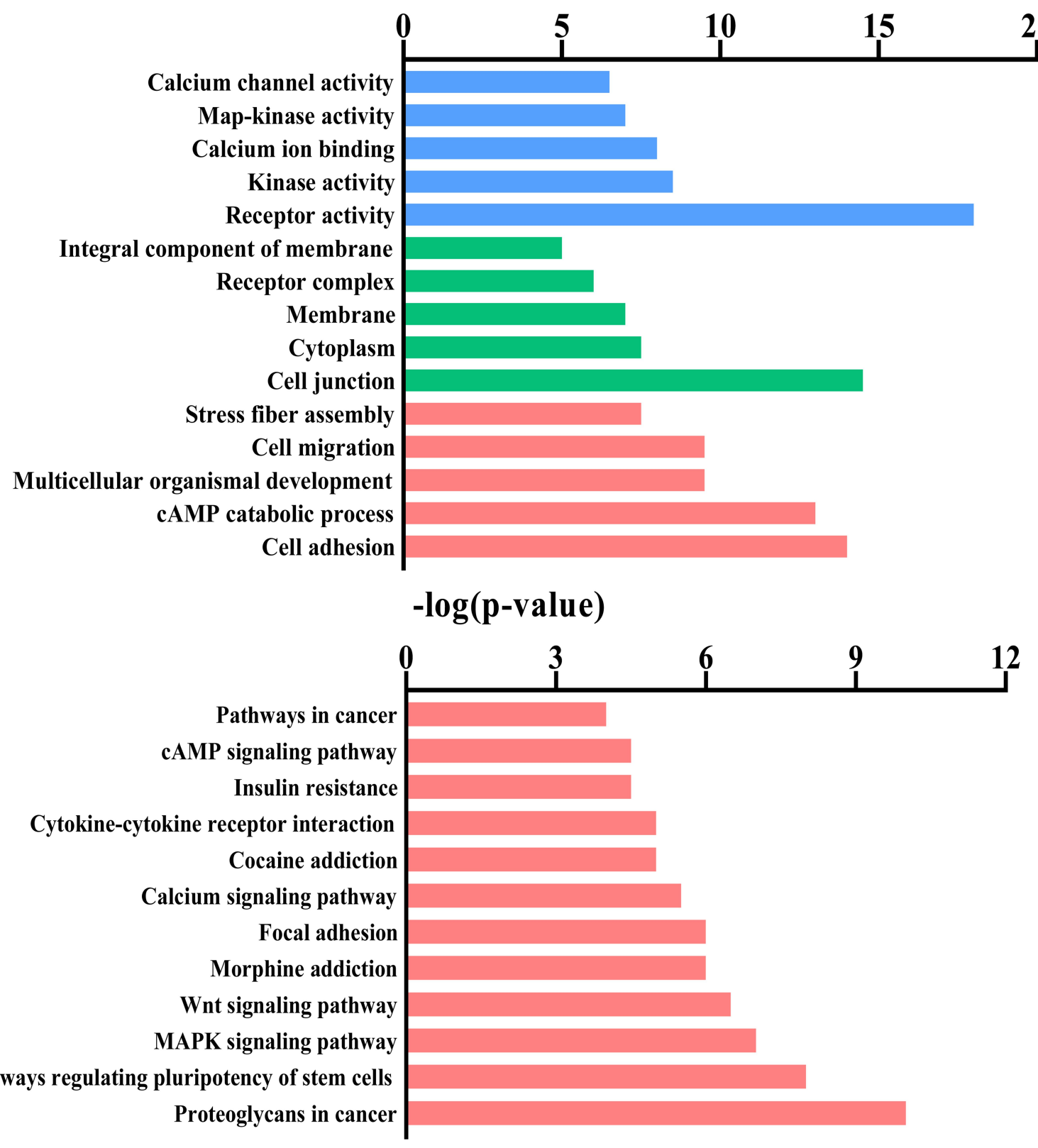

Figure 2 GO and KEGG enrichment analysis based on the target genes of differentially expressed tRFs between GC tissues and adjacent tissues. (A) Gene Ontology (GO) analysis of differentially expressed tRFs target genes in GC tissues and adjacent tissues. (B) Top I 2 Kyoto Encyclopedia of Genes and Genomes (KEGG) pathways of tRFs candidate target genes in GC tissues and adjacent tissues. 


\section{Expression Levels of tRF-24-V29K9UV3IU in GC Tissues and Cell Lines}

Considering the uncertainty of high-throughput sequencing, real-time PCR was used to validate tRF-21-2N37WX01B, tRF-30-FQR47673FEWS, tRF-28-P4R8YP9LOND5, tRF24-V29K9UV3IU, and tRF-22-8E89YZB52 with high fold differences and high expression levels in $10 \mathrm{GC}$ tissues and adjacent tissues. tRF-22-8E89YZB52, tRF-212N37WX01B, and tRF-24-V29K9UV3IU were found to be significantly downregulated in cancer tissues, which is consistent with the sequencing results (Figure 3A). The expression of these three tRFs was detected in three different GC cells (BGC-823, SGC-7907, MKN-45), respectively (Figure 3B). BGC-823 cells showed the lowest tRF expression. Hence, BGC-823 was included in the follow-up studies.

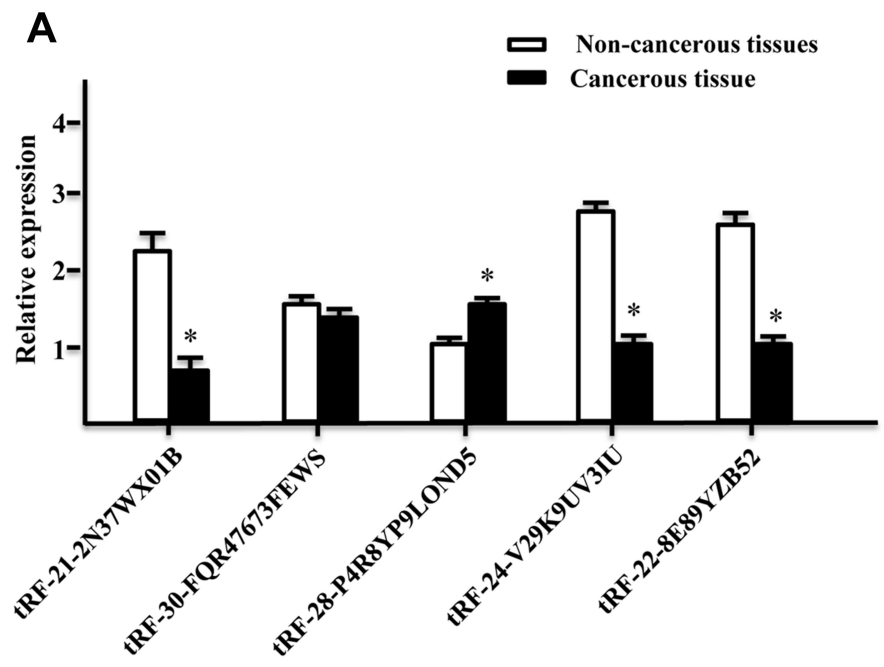

C

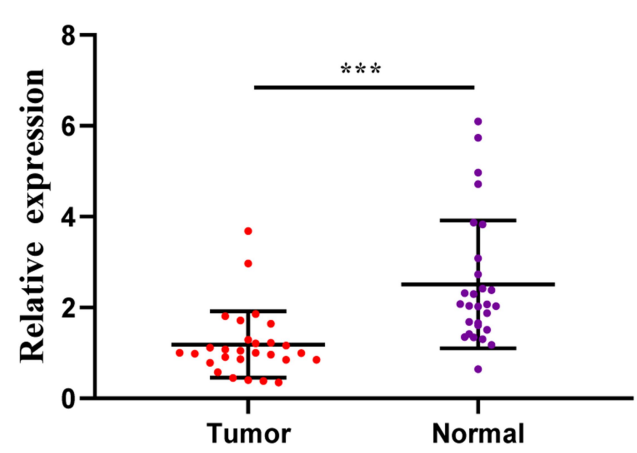

We further verified the expression of tRF-24V29K9UV3IU in 28 pairs of GC tissues and adjacent tissues by qRT-PCR experiments, and found that it was significantly downregulated in GC tissues (Figure 3C).

To determine the diagnostic potential of tRF-24V29K9UV3IU in $\mathrm{GC}$, receiver operating characteristic (ROC) curves were constructed in GC tissues $(n=28)$ and adjacent tissues $(\mathrm{n}=28)$ (Figure 3D). The ROC curve for the expression levels of tRF-24-V29K9UV3IU in GC and paracancer tissues had an AUC of 0.8712 (95\% confidence interval: 77.35\% 98.73\%) (Figure 3D). In addition, the optimal cutoff value for tRF-24-V29K9UV3IU expression was 1.297 in GC. Accordingly, expression levels less than 1.297 had a sensitivity of $78.57 \%$ and a specificity of $92.86 \%$. The ROC curve results suggest that tRF-24-V29K9UV3IU may be used as a diagnostic biomarker for gastric cancer.

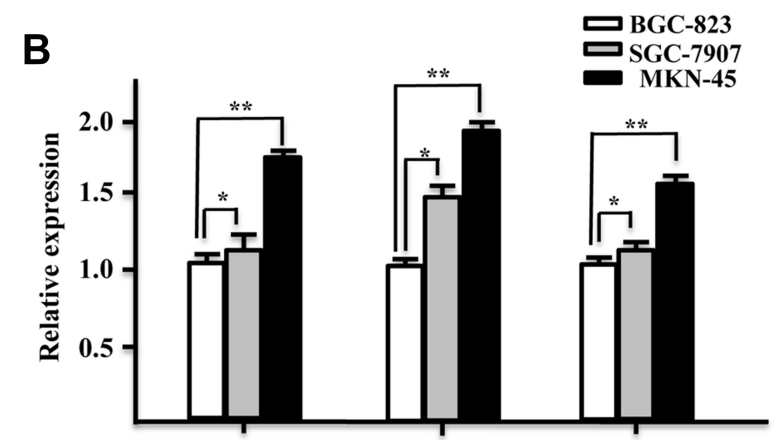

tRF-21-2N37WX01B tRF-24-V29K9UV3IU tRF-22-8E89YZB52

D

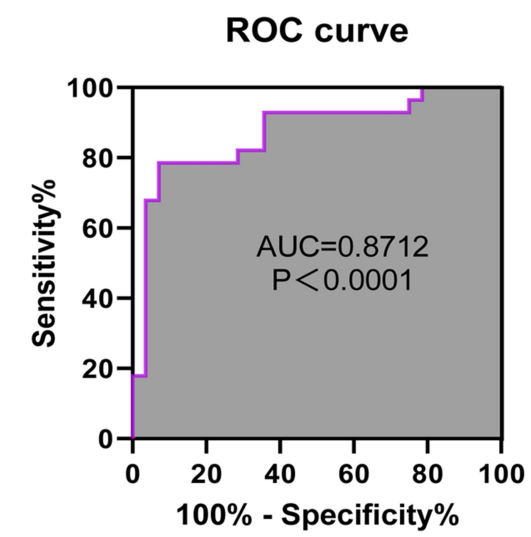

Figure 3 Detection of differentially expressed tRFs between GC tissues and adjacent tissues, and GC cell lines. (A) The 5 differentially expressed tRFs were verified by qRTPCR between GC tissues and adjacent tissues. (B) The expressions of tRF-2I-2N37WX0IB, tRF-22-8E89YZB5, and tRF-24-V29K9UV3IU were verified by qRT-PCR in three different GC cell lines. (C) The expression of tRF-24-V29K9UV3IU was assessed by qRT-PCR between 28 pairs of GC tissues and adjacent tissues. (Each dot represents a sample.) (D) The diagnostic potential of tRF-24-V29K9UV3IU was assessed with a ROC curve in 28 pairs of GC tissues and paracancer tissues. (*P <0.05; ** P <0.0I; ***P $<0.001$, compared with the paracancerous group). 

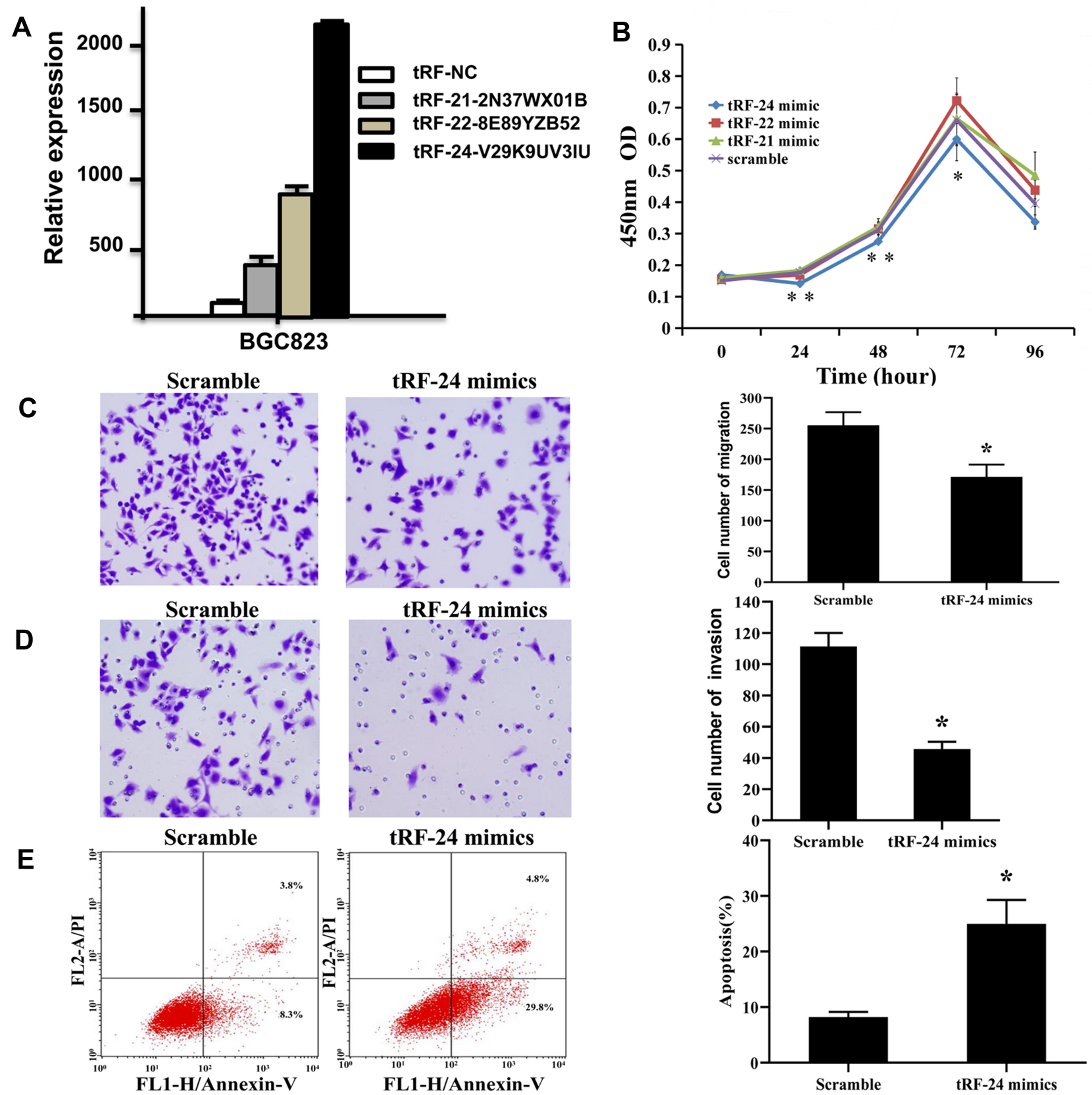

E
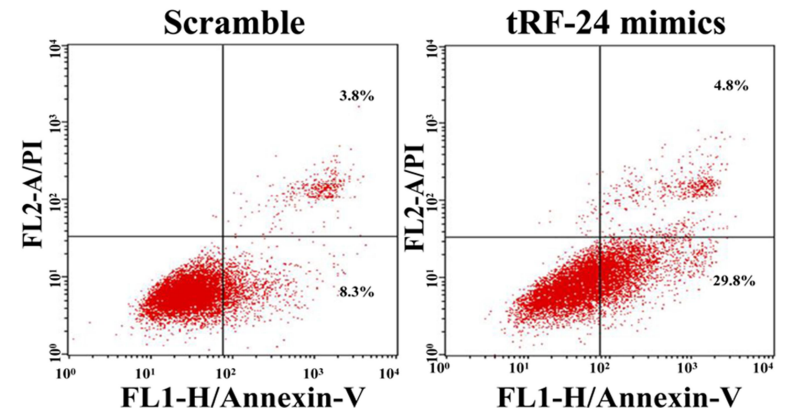
apoptosis of MKN-45 cells, compared with the control group (Figure $5 \mathrm{~A}-\mathrm{D})$.

\section{Construction of the tRF-24-V29K9UV3IU Target Gene Network}

The functions of tRFs are similar to those of miRNAs, and this may be related to the regulatory target gene expression in GC. Therefore, we further analyzed the target gene of tRF-24-V29K9UV3IU and constructed a target gene network (Figure 6). The results showed that tRF-24-V29K9UV3IU can target many genes associated with classical signaling pathways, which are involved in cancer development and metastasis, such as ErbB (ErbB2), MAPK (MAP3K7, MAP2K3, MAPK8IP1, MAPK8IP2, MAPK8IP3, etc.), Wnt/ $\beta$ Catenin (WNT4, WNT10A, WNT3A, etc.), and chemokine (CXCR5, CXCR3, CX3CL1, CX3CR1, CXCL9, etc.) signaling pathways.

\section{Validation of the Target mRNAs}

In order to screen the target genes of tRF-24-V29K9UV3IU, we analyzed the 3 tumor-related signaling pathways, including MAPK, Wnt, and chemokine signaling pathways. We found that the Wnt signaling pathway was regulated by 7 tRFs (tRF-30-XQR47673FEWS, tRF-30-P4R8YP9LON4V, tRF-30-FQR47673FEWS, tRF-29-P4R8YP9LONHK, tR F-24-V29K9UV3IU, tRF-28-P4R8YP9LOND5, tRF22-8E89YZB52) simultaneously, while the MAPK and chemokine signaling pathways were regulated by only 6 differentially expressed tRFs. In addition, among the molecules on the Wnt pathway targeted by tRF-24V29K9UV3IU, CCND2, DAAM2, FZD3, VANGL1, and WNT3A can be regulated by other tRFs simultaneously. Therefore, we selected these 5 genes as the candidate target genes of tRF-24-V29K9UV3IU. qRT-PCR verification showed that the expressions of CCND2, FZD3, and VANGL1 were significantly decreased in MKN-45 cells transfected with tRF-24 mimics (Figure 7). The target genes DAAM2 and WNT3A were not statistically significant between the two groups. These data suggested that tRF-24V29K9UV3IU played a significant role in the Wnt pathway by regulating CCND2, FZD3, and VANGL1.

\section{Discussion}

GC is one of the common malignant digestive tract tumors, which ranks second in cancer mortality. ${ }^{1}$ With the increasing incidence of $\mathrm{GC}$ in younger people, the invasion, metastasis, and postoperative recurrence of GC seriously endanger the health of Chinese residents. ${ }^{18}$ Several studies have confirmed that tRFs are key regulatory factors in tumor-associated processes, and tRFs may become new diagnostic and therapeutic targets for cancer therapy. ${ }^{19}$ Many tRFs have distinct biological functions: they act as tumor suppressors, oncogenes, or regulators of protein synthesis, or they induce apoptosis or neurodegeneration. ${ }^{7}$ However, they regulate the mechanism of telomere restriction fragments in gastric mucosal carcinogenesis process. This study is the first to comprehensively evaluate the roles of tRFs in GC. Differential expressions of eight tRFs were detected in GC tissues and adjacent tissues. Real-time PCR confirmed that the expression level of tRF-24-V29K9UV3IU in GC patients was significantly decreased compared with that in the adjacent tissues. Further studies showed that tRF-24-V29K9UV3IU significantly inhibited GC cell proliferation, migration, and invasion, and promoted cell apoptosis. tRFs play important roles in cellular processes and are involved in multiple cancers. For example, mice overexpressing the TRF2 telomere-binding protein K5-TRF2 showed premature aging and increased cancer severity in the presence of telomerase activity. $^{20}$ The expression level of TRF1 protein is significantly decreased in kidney cancer, and it is correlated with the malignant degree of the cancer. ${ }^{21}$ To date, no study has reported the specific function of tRF-24-V29K9UV3IU. Our study showed that the expression of tRF-24V29K9UV3IU in GC tissues and GC cell lines was significantly lower than that in GC paracancerous cells. Importantly, overexpression of tRF-24-V29K9UV3IU in GC cell BGC-823 significantly inhibited cell proliferation, migration, and invasion, while promoting cell apoptosis. Therefore, tRF-24-V29K9UV3IU might act as a cancer suppressor for GC and a novel target for GC therapy.

MAPK was significantly enriched by tRF-24V29K9UV3IU. In addition, the P38 MAPK signaling pathway is involved in key processes of tumor progression such as invasion, inflammation, and angiogenesis. ${ }^{22}$ Moreover, tRF-24-V29K9UV3IU targets Wnt/ $\beta$-catenin. The $\mathrm{Wnt} / \beta$-catenin signaling pathway regulates many important biological processes, including cell migration, polarization, morphogenesis, and the growth of organs during embryonic development. ${ }^{23}$ Classical Wnt signaling pathway mediates important cell cancer occurrence. Therefore, we can inhibit the metastasis of GC cells by targeting the $\mathrm{Wnt} / \beta$-catenin and MAPK signaling pathways. Additionally, tRF-24-V29K9UV3IU can target 


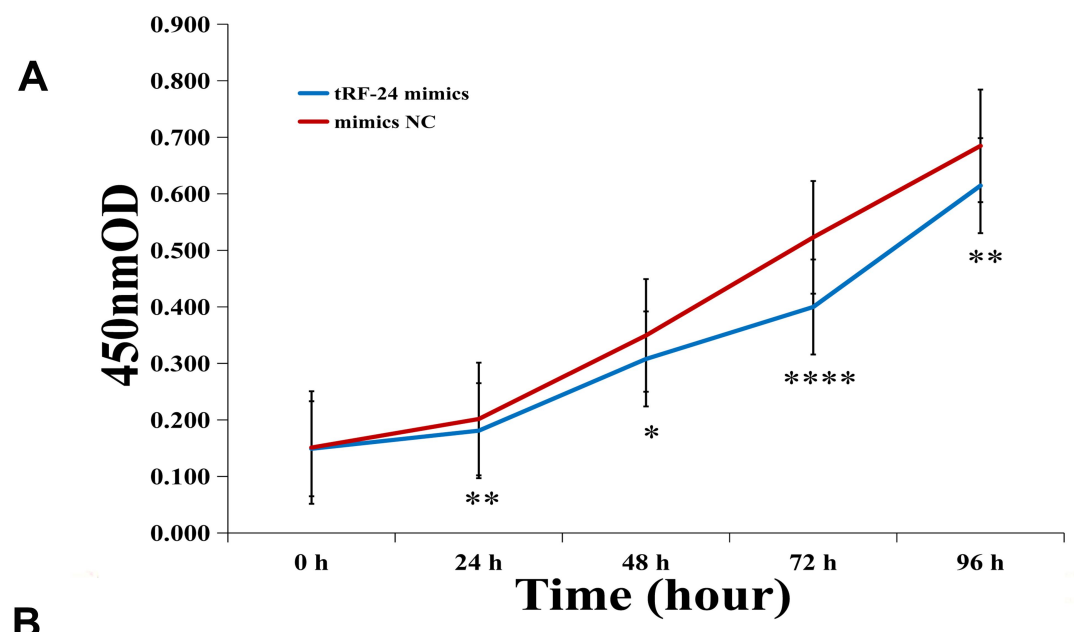

B

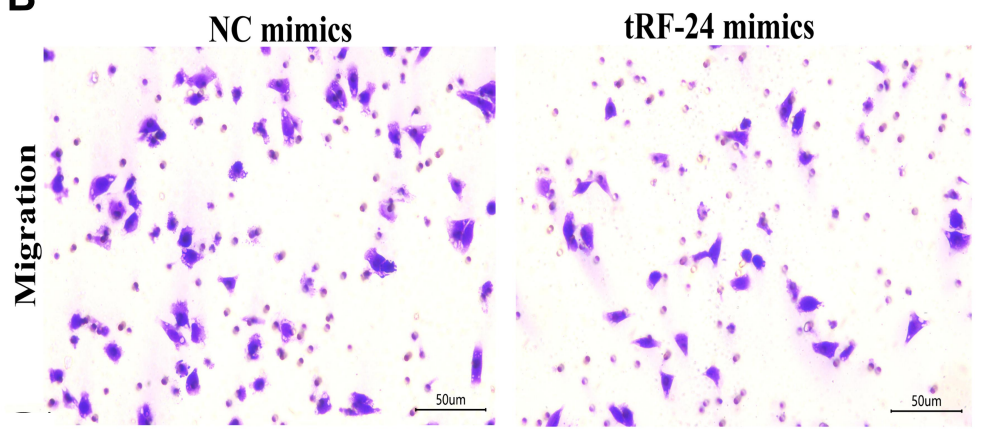

C

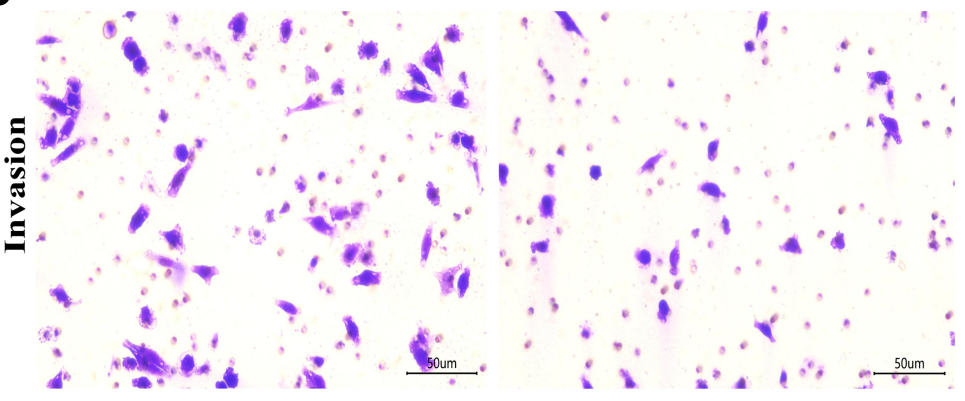

D
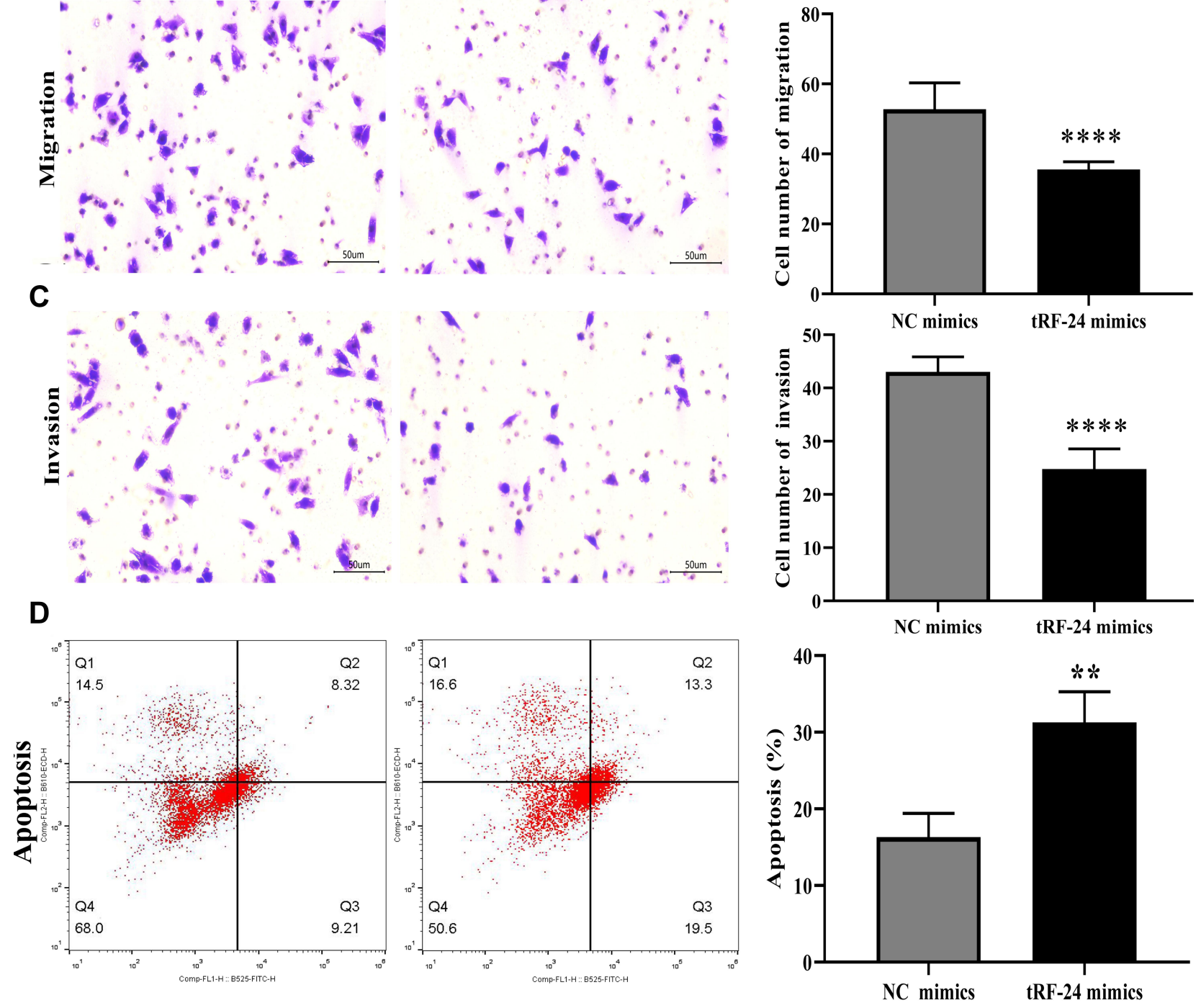

Figure 5 tRF-24-V29K9UV3IU mimics inhibited MKN-45 cell proliferation, invasion, migration, while enhancing MKN-45 cell apoptosis. (A) The proliferation ability of MKN-45 cells was analyzed by CCK-8. (B and C) The migration and invasion abilities of MKN-45 cells were analyzed by transwell. Three random fields under the microscope were taken for counts and statistical results. (D) The apoptosis rate in MKN-45 cells was determined by flow cytometry analysis. Asterisk indicates significant differences between groups ( $* \mathrm{P}<0.05 ; * * \mathrm{P}<0.01$; $* * * * \mathrm{P}<0.000 \mathrm{I})$. 


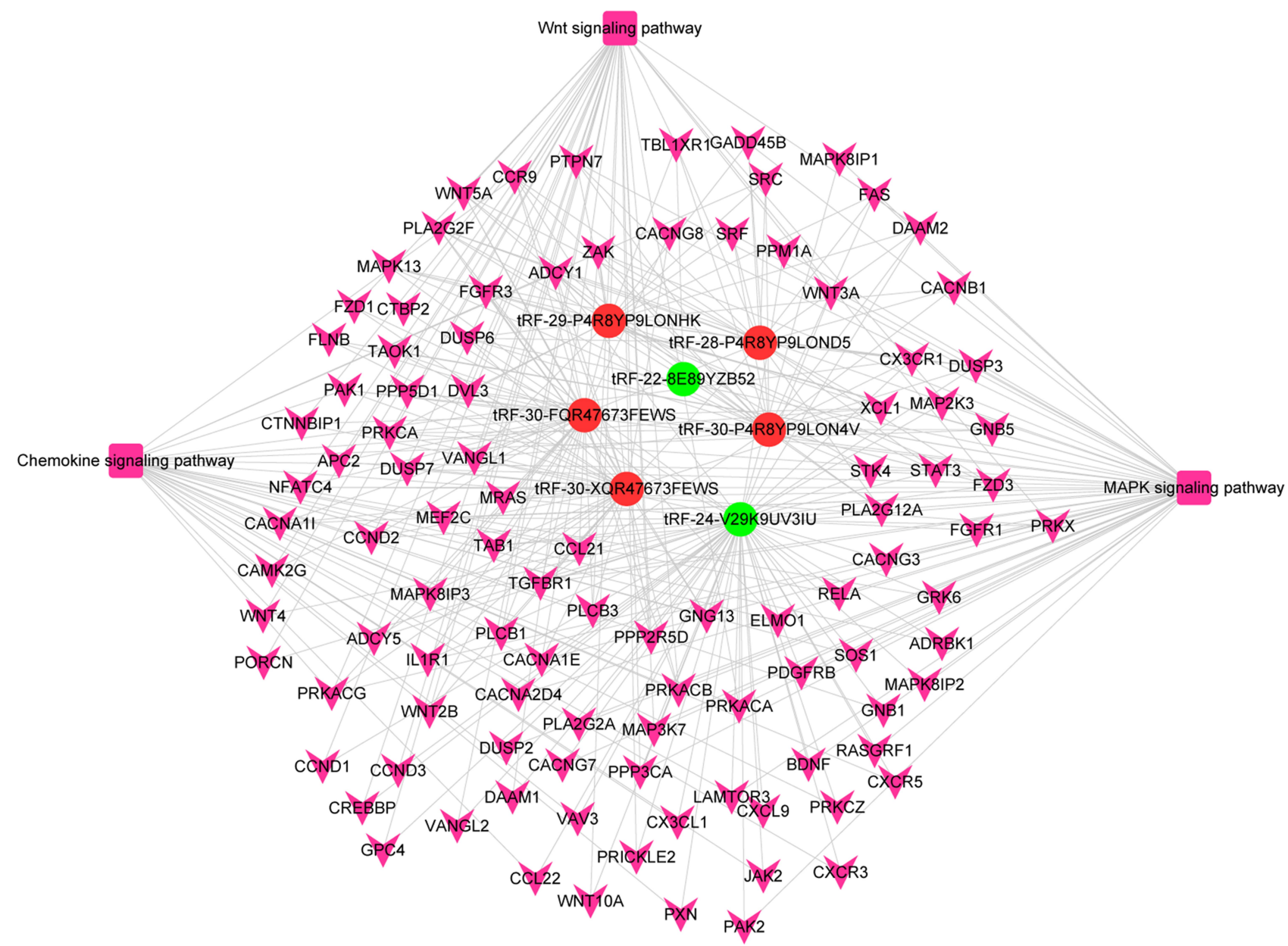

Figure 6 The network of the tRF-mRNA-pathway constructed with 7 selected tRFs ( 5 were upregulated and, 2 were downregulated) and their target gene. Note: Circle indicates the key tRFs, red represents upregulation, and green represents downregulation; $V$ illustrates the target gene that may be regulated; Quadrate indicates the pathway.

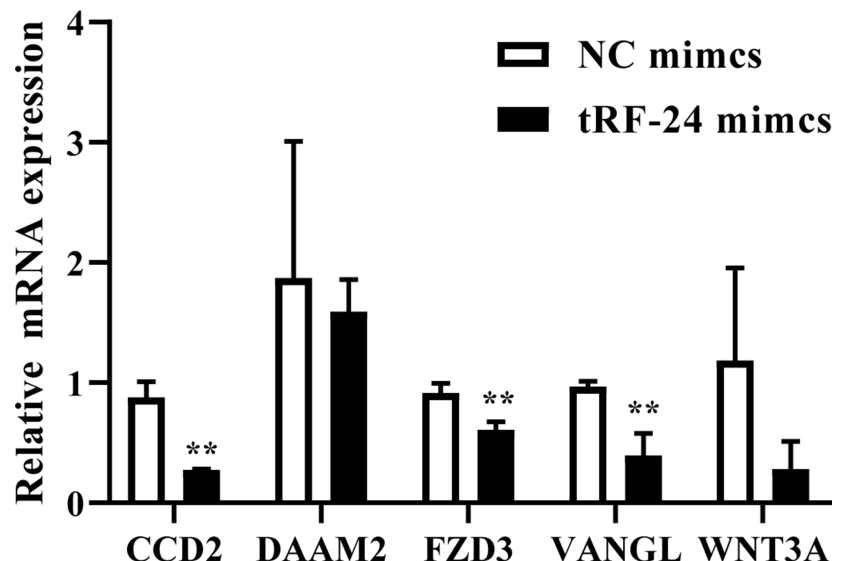

Figure 7 Verification of the target gene of tRF-24-V29K9UV3IU on wingless-type(Wnt) signaling pathway. Histogram indicates that target genes CCND2, FZD3, VANGLI of tRF-24-V29K9UV3IU were significantly down-regulated in the MKN-45 cells with tRF-24-V29K9UV3IU mimics. (**P<0.0I).

a variety of chemokines. Chemokines, when bound to their receptors, play an important role in lymphoid organs, lymphoid circulation, Th1/Th2 cell differentiation, and inflammation. In breast cancer cells, actin polymerization and pseudopodia formation are mediated by CXCR4 or CCR7 signaling pathways, and they subsequently induce chemotactic and invasive responses. ${ }^{24}$ It is suspected that tRF-24-V29K9UV3IU may participate in the development of GC through these pathways and may be involved in the occurrence and metastasis of many cancers.

\section{Conclusions}

In conclusion, tRF-24-V29K9UV3IU may inhibit cell proliferation, migration, and invasion, while promoting apoptosis by regulating the $\mathrm{Wnt} / \beta$-catenin, MAPK, and $\mathrm{Wnt}$ signaling pathways. tRF-24-V29K9UV3IU may provide a novel diagnostic and therapeutic option for patients with GC. 


\section{Data Sharing Statement}

The datasets used and/or analyzed in this study are available from the corresponding author upon reasonable request.

\section{Ethics Approval and Consent to Participate}

This study was approved by the ethics committee of Kunming Medical University.

\section{Patient Consent for Publication}

All patients provided written informed consent.

\section{Author Contributions}

All authors made substantial contributions to the conception and design, acquisition of data, or analysis and interpretation of data; took part in drafting the article or revising it critically for important intellectual content; agreed on the journal to which the article will be submitted; gave final approval of the version to be published; and agreed to be accountable for all aspects of the work.

\section{Funding}

This work was supported by the National Natural Science Foundation of China (81860424).

\section{Disclosure}

The authors report no conflicts of interest in this work.

\section{References}

1. Chen W, Zheng R, Baade PD, et al. Cancer statistics in China, 2015. CA Cancer J Clin. 2016;66(2):115-132. doi:10.3322/caac.21338

2. Song $\mathrm{Z}, \mathrm{Wu} \mathrm{Y}$, Yang J, et al. Progress in the treatment of advanced gastric cancer. Tumour Biol. 2017;39(7):1010428317714626. doi: $10.1177 / 1010428317714626$

3. Roy RK, Hoppe MM, Srivastava S, et al. CEACAM6 is upregulated by helicobacter pylori $\mathrm{CagA}$ and is a biomarker for early gastric cancer. Oncotarget. 2016;7(34):55290-55301. doi:10.18632/ oncotarget. 10528

4. Lee YS, Shibata Y, Malhotra A, et al. A novel class of small RNAs. tRNA-derived RNA fragments (tRFs). Genes Dev. 2009;23 (22):2639-2649. doi:10.1101/gad.1837609

5. Maute RL, Schneider C, Sumazin P, et al. tRNA-derived microRNA modulates proliferation and the DNA damage response and is down-regulated in B cell lymphoma. Proc Natl Acad Sci U S A. 2013;110(4):1404-1409. doi:10.1073/pnas.1206761110

6. Yamasaki S, Ivanov P, Hu GF, et al. Angiogenin cleaves tRNA and promotes stress-induced translational repression. J Cell Biol. 2009;185 (1):35-42. doi:10.1083/jcb.200811106
7. Kumar P, Kuscu C, Dutta A. Biogenesis and function of transfer RNA-related fragments (tRFs). Trends Biochem Sci. 2016;41 (8):679-689. doi:10.1016/j.tibs.2016.05.004

8. Mishima E, Inoue C, Saigusa D, et al. Conformational change in transfer RNA is an early indicator of acute cellular damage. J Am Soc Nephrol. 2014;25(10):2316-2326. doi:10.1681/ASN.2013091001

9. Wang Q, Lee I, Ren J, et al. Identification and functional characterization of tRNA-derived RNA fragments (tRFs) in respiratory syncytial virus infection. Mol Ther. 2013;21(2):368-379. doi:10.1038/ mt.2012.237

10. Park EJ, Kim TH. Fine-tuning of gene expression by tRNA-derived fragments during abiotic stress signal transduction. Int $J \mathrm{Mol} \mathrm{Sci}$. 2018;19(2):e518. doi:10.3390/ijms19020518

11. Kim HK, Fuchs G, Wang S, et al. A transfer-RNA-derived small RNA regulates ribosome biogenesis. Nature. 2017;552(7683):57-62. doi:10.1038/nature25005

12. Anderson P, Ivanov P. tRNA fragments in human health and disease. FEBS Lett. 2014;588(23):4297-4304. doi:10.1016/j. febslet.2014.09.001

13. Balatti V, Nigita G, Veneziano D, et al. tsRNA signatures in cancer. Proc Natl Acad Sci U S A. 2017;114(30):8071-8076. doi:10.1073/ pnas. 1706908114

14. Olvedy M, Scaravilli M, Hoogstrate Y, et al. A comprehensive repertoire of tRNA-derived fragments in prostate cancer. Oncotarget. 2016;7(17):24766-24777. doi:10.18632/oncotarget.8293

15. Zhou K, Diebel KW, Holy J, et al. A tRNA fragment, tRF5-Glu, regulates BCAR3 expression and proliferation in ovarian cancer cells. Oncotarget. 2017;8(56):95377-95391. doi:10.18632/ oncotarget.20709

16. Huang B, Yang $\mathrm{H}$, Cheng $\mathrm{X}$, et al. tRF/miR-1280 suppresses stem cell-like cells and metastasis in colorectal cancer. Cancer Res. 2017;77(12):3194-3206. doi:10.1158/0008-5472.CAN-16-3146

17. Goodarzi H, Liu X, Nguyen HC, et al. Endogenous tRNA-derived fragments suppress breast cancer progression via YBX1 displacement. Cell. 2015;161(4):790-802. doi:10.1016/j. cell.2015.02.053

18. Wang P, Sun Z, Wang W, et al. Conditional survival of patients with gastric cancer who undergo curative resection: a multi-institutional analysis in China. Cancer. 2018;124(5):916-924. doi:10.1002/ cncr.31160

19. Cui Y, Huang Y, Wu X, et al. Hypoxia-induced tRNA-derived fragments, novel regulatory factor for doxorubicin resistance in triple-negative breast cancer. $J$ Cell Physiol. 2019;234 (6):8740-8751. doi:10.1002/jcp.27533

20. Munoz P, Blanco R, Blasco MA. Role of the TRF2 telomeric protein in cancer and ageing. Cell Cycle. 2006;5(7):718-721. doi:10.4161/ cc.5.7.2636

21. Shi JM, Ding W, Huang H, et al. Expression of telomere repeat binding factor 1 (TRF1) protein in kidney cancer. Zhejiang Da Xue Xиe Bao Yi Xue Ban. 2004;33:496-499.

22. Wagner EF, Nebreda AR. Signal integration by JNK and p38 MAPK pathways in cancer development. Nat Rev Cancer. 2009;9 (8):537-549. doi:10.1038/nrc2694

23. Kwak HJ, Park DW, Seo JY, et al. The Wnt/ß-catenin signaling pathway regulates the development of airway remodeling in patients with asthma. Exp Mol Med. 2015;47(12):e198. doi:10.1038/ emm.2015.91

24. Müller A, Homey B, Soto $\mathrm{H}$, et al. Involvement of chemokine receptors in breast cancer metastasis. Nature. 2001;410 (6824):50-56. doi:10.1038/35065016 


\section{Publish your work in this journal}

OncoTargets and Therapy is an international, peer-reviewed, open access journal focusing on the pathological basis of all cancers, potential targets for therapy and treatment protocols employed to improve the management of cancer patients. The journal also focuses on the impact of management programs and new therapeutic agents and protocols on patient perspectives such as quality of life, adherence and satisfaction. The manuscript management system is completely online and includes a very quick and fair peer-review system, which is all easy to use. Visit http://www.dovepress.com/ testimonials.php to read real quotes from published authors.

Submit your manuscript here: https://www.dovepress.com/oncotargets-and-therapy-journal 